\title{
The role of nuclear factor of activated T cells during phorbol myristate acetate-induced cardiac differentiation of mesenchymal stem cells
}

Hyang-Hee Seo ${ }^{1}$, Chang Youn Lee ${ }^{2}$, Jiyun Lee ${ }^{1}$, Soyeon Lim³ ${ }^{3}$ Eunhyun Choi ${ }^{3}$, Jong-Chul Park', Seahyoung Lee Le, $^{3, *}$ and Ki-Chul Hwang ${ }^{3,5^{*}}$

\begin{abstract}
Background: We previously reported that phorbol 12-myristate 13-acetate (PMA) treatment can induce the cardiac differentiation of mesenchymal stem cells (MSCs). In the present study, we investigated how PMA induces cardiac differentiation of MSCs, focusing on its effect on the transcription factors responsible for increased cardiac marker gene expression.

Methods: Human MSCs (hMSCs) were treated with $1 \mu$ M PMA for 9 days. The expression of MSC markers and cardiac markers in the PMA-treated hMSC, as well as the nuclear translocation of transcription factors, nuclear factor of activated T cells (NFAT), and myogenic differentiation 1 (MyoD), was examined. Transcriptional activity of NFAT was examined by utilizing a green fluorescent protein (GFP) vector containing NFAT motif of human interleukin-2 promoter. The effect of PMA on the expression of key cell cycle regulators was examined.

Results: PMA induces the transcriptional activity of NFAT and MyoD, which have been associated with increased expression of cardiac troponin $\mathrm{T}$ (cTnT) and myosin heavy chain $(\mathrm{MHC})$, respectively. Our data suggested that protein kinase C (PKC) mediates the effect of PMA on NFAT activation. Furthermore, PMA treatment increased cell-cycle regulator p $27^{\text {kip } 1}$ expression, suggesting that PMA triggers the cardiac differentiation program in MSCs by regulating key transcription factors and cell cycle regulators.

Conclusions: The results of this study demonstrate the importance of NFAT activation during PMA-induced MSC differentiation and help us to better understand the underlying mechanisms of small molecule-mediated MSC differentiation so that we can develop a strategy for synthesizing novel and improved differentiation-inducing small molecules.
\end{abstract}

Keywords: Phorbol myristate acetate, MSC, NFAT, Small molecule, Cardiac differentiation

\section{Introduction}

One common stem cell-based therapy involves the in vitro expansion of stem cells to acquire a sufficient number of cells and the re-introduction of these cells into damaged tissues [1]. However, certain tissues, such as the myocardium, require more than just a high number of therapeutic cells. For example, unsynchronized

\footnotetext{
* Correspondence: sam1017@ish.ac.kr; kchwang@cku.ac.kr

${ }^{3}$ Institute for Bio-medical Convergence, Catholic Kwandong University, Incheon, South Korea

Full list of author information is available at the end of the article
}

electric coupling between the transplanted cells and cardiomyocytes can increase the proarrhythmic potential [2]; therefore, therapeutic cells resembling native cardiomyocytes would be preferred in terms of their functionality after transplantation compared with undifferentiated stem cells [3]. One method for inducing differentiation of stem cells into a desired lineage is small molecule treatment.

To date, various small molecules with different biological mechanisms have been utilized to induce the cardiac differentiation of stem cells. For example, a specific 
p38 mitogen-activated protein kinase (MAPK) inhibitor [4] and a bone morphogenetic protein (BMP) inhibitor [5] induced the cardiac differentiation of embryonic stem cells. Furthermore, several studies have demonstrated that a number of small molecule inhibitors of Wnt signaling promoted cardiomyogenesis in stem cells [6-8]. We have also reported that phorbol 12-myristate 13-acetate (PMA), a well-known protein kinase C (PKC) activator, induced the expression of cardiac marker genes such as myosin heavy chain (MHC) and cardiac troponin $\mathrm{T}$ (cTnT) in mesenchymal stem cells (MSCs), thus demonstrating its potential as a cardiac differentiation inducer [9]. Nevertheless, that particular previous study lacked detailed explanations of the underlying mechanisms of how PMA induces cardiac differentiation in MSCs.

Since the small molecule-mediated regulation of protein expression is what renders the phenotypic change toward a desired lineage of cells, it is reasonable to hypothesize that PMA activates certain transcription factors that drive the expression of cardiac marker genes in MSCs. Thus, in the present study, we investigated the effect of PMA on the activation of key transcription factors to further clarify the underlying molecular mechanism of PMA treatment during MSC differentiation.

The transcription factor myogenic differentiation 1 (MyoD) is an early marker of myogenic differentiation and is known to regulate the expression of MHC [10]. MyoD has been reported to convert a variety of cells, such as nerve, liver, and fat cells, to skeletal muscle, demonstrating that it is a powerful master regulator of myogenic differentiation [11]. Because PKC has been reported to be required for $\beta$-catenin-indepenent activation of MyoD [12], it is possible that PMA-induced activation of PKC subsequently enhances the transcriptional activity of MyoD and results in increased MHC expression in MSCs. Another cardiac marker, cTnT, is reportedly regulated by nuclear factor of activated $\mathrm{T}$ cells (NFAT) [13]. Similar to MyoD, the transcriptional activity of NFAT can also be regulated by PKC-mediated phosphorylation [14]. Thus, the reported cardiac differentiation ability of PMA may involve activation of these transcription factors, namely MyoD and NFAT, and we examined such a possibility in the present study. We investigated the effect of PMA on the activation status of these transcription factors and its related mechanisms.

\section{Materials and methods}

\section{Induction of cardiogenic differentiation}

Human MSCs (hMSC; Lonza, Allendale, NJ, USA) were seeded in $60-\mathrm{mm}$ cell culture plates at a density of $2 \times 10^{5}$ cells/plate, and PMA (Sigma-Aldrich Corp., St. Louis, MO, USA) was added to a final concentration of $1 \mu \mathrm{M}$. PMA powder was reconstituted in ethanol to make $1 \mathrm{mM}$ PMA stock solution. For treatment, 1 mM PMA stock was 1:1000 diluted in culture medium. hMSCs were cultured in DMEM-low glucose (Thermo Fisher Scientific, Pittsburgh, PA, USA) supplemented with $10 \%$ fetal bovine serum. The medium was replaced every 3 days with PMA treatment for 9 days.

\section{Immunofluorescence}

Cells were cultured in four-well slide chambers. The cells were permeabilized using $0.1 \%$ Triton X-100 for $10 \mathrm{~min}$. Next, the cells were blocked for $1 \mathrm{~h}$ in a blocking solution ( $2 \%$ bovine serum albumin and $10 \%$ horse serum in phosphate-buffered saline) and incubated with cTnT (ab8295; Abcam, Cambridge, MA, USA), $\beta$-MHC (ab15; Abcam) and CD90 antibodies (sc-6071; Santa Cruz Biotechnology, Dallas, TX, USA). FITC-conjugated mouse, rabbit, and goat secondary antibodies (Jackson ImmunoResearch Laboratories, West Grove, PA, USA) were used. Immunofluorescence was detected by confocal microscopy (LSM710; Carl Zeiss Microscopy GmbH, Jena, Germany).

\section{Co-localization image analysis}

The degree of co-localization was measured by ZEN 2009 Light Edition software that calculated the percentage of co-localized pixels relative to all pixels obtained. Following Carl Zeiss Micro-imaging, the Pearson's correlation coefficient R (PCC) value was calculated. The value for PCC can range from -1 to 1 , and a value of 1 indicates that the fluorescence patterns of the two molecules are perfectly matched [15].

\section{Western blot assay}

Equal amounts of proteins were separated by SDSPAGE. After blocking the membrane with $5 \%$ skim milk for $1 \mathrm{~h}$ at room temperature, the membranes were incubated with primary antibodies against NFAT (sc271127), MyoD (sc-760), p-MARCKS (sc-101730), and p27 (sc-528; all Santa Cruz Biotechnology), LaminB2 (sab2702205; Thermo Fisher Scientific), and $\beta$-actin (A5316; Sigma-Aldrich Corp.) overnight at $4{ }^{\circ} \mathrm{C}$. The membrane was washed and incubated for $1 \mathrm{~h}$ at room temperature with horseradish peroxidase-conjugated secondary antibodies. The bands were detected by an enhanced chemiluminescence (ECL) reagent (Santa Cruz Biotechnology). The band intensities were quantified using NIH Image J version $1.34 \mathrm{e}$ software.

\section{Nuclear and cytoplasmic extraction}

For nuclear and cytoplasmic extraction, NE-PER Nuclear and Cytoplasmic Extraction Reagents (Thermo Fisher Scientific) were used according to the user manual provided by the manufacturer. 


\section{Immunoprecipitation}

For immunoprecipitation (IP), cells were lysed in IP lysis buffer (Thermo Fisher Scientific) for $30 \mathrm{~min}$ on ice. Cell lysates were centrifuged at 10,000 g for $20 \mathrm{~min}$ and the supernatant was retained. After protein quantification, the lysates were incubated with calcineurin A antibody (ab3673; Abcam), and the tubes were rotated for $1 \mathrm{~h}$ at $4{ }^{\circ} \mathrm{C}$. After $1 \mathrm{~h}$, Protein G Dynabeads (Thermo Fisher Scientific) were added to the lysates and incubated overnight at $4{ }^{\circ} \mathrm{C}$. The Dynabeads-antibody-calcineurin $\mathrm{A}$ complexes were centrifuged at $2500 \mathrm{~g}$ for $30 \mathrm{~min}$ at $4{ }^{\circ} \mathrm{C}$, and the antibody-calcineurin A complexes were eluted with elution buffer, and the eluted proteins were heated for $10 \mathrm{~min}$ at $99^{\circ} \mathrm{C}$ and subjected to SDS-PAGE electrophoresis.

\section{Construction of the pAc-NFAT-GFP vector and transfection}

To construct a green fluorescent protein (GFP) vector containing the NFAT binding motif of human interleukin-2 (GGAGGAAAAACTGTTTCATACAGAA GGCGT, corresponding to $-286 /-257$ of the human interleukin-2 promoter [16]), two copies of the NFAT binding motif were synthesized and cloned into the pAcGFP1-1 (Clontech, Mountain View, CA, USA) vector using HindIII and BamHI enzymes. The constructed GFP vectors were delivered to the cells using Lipofectamine LTX (Thermo Fisher Scientific). For six-well plates, $500 \mathrm{ng} /$ well of each vector was used.

\section{RT-PCR}

The expression levels of various genes were analyzed by reverse transcription polymerase chain reaction (RTPCR). Complementary DNA (cDNA) was generated using 500 ng of total RNA with the Promega Reverse Transcription System according to the manufacturer's instructions. The PCR conditions consisted of denaturing at $94{ }^{\circ} \mathrm{C}$ for $3 \mathrm{~min}$, followed by 35 cycles of denaturation at $94{ }^{\circ} \mathrm{C}$ for $30 \mathrm{~s}$, annealing at $94{ }^{\circ} \mathrm{C}$ for $30 \mathrm{~s}$, and extension at $72{ }^{\circ} \mathrm{C}$ for $30 \mathrm{~s}$, before a final extension at $72{ }^{\circ} \mathrm{C}$ for $10 \mathrm{~min}$. RT-PCR products were separated by electrophoresis on a $1.2 \%$ agarose gel (Bio-Rad Laboratories, Inc., Hercules, CA, USA). Glyceraldehyde3 -phosphate dehydrogenase (GAPDH) was used as an internal standard.

\section{Statistical analysis}

Quantitative data were expressed as the means \pm SD of at least three independent experiments. For statistical analysis, one-way analysis of variance (ANOVA) with Bonferroni correction was performed using OriginPro 8 SR4 software (ver. 8.0951, OriginLab Corporation, Northampton, MA, USA) if there were more than three groups. A $p$ value of less than 0.05 was considered statistically significant.

\section{Result \\ PMA-induced cardiac marker expression in hMSCs}

The cells were treated with PMA $(1 \mu \mathrm{M})$ for 9 days based on a previous report in which the same regime induced the cardiac differentiation of rMSCs [9]. After 9 days of PMA treatment, the expression levels of the cardiac-specific markers cTnT and MHC increased in the PMA-treated group compared with those of the untreated control (Fig. 1a, b). On the other hand, the expression of the stem cell marker CD90 decreased with PMA treatment compared with that of the untreated control (Fig. 1c). Additionally, the RT-PCR result indicated that PMA increased the expression of both $\mathrm{MHC}$ and cTnT at the RNA level compared to control (Fig. 1d). These data suggested that PMA treatment induced the differentiation of hMSCs into cells with cardiomyocyte characteristics.

\section{PMA induced activation of the transcription factors NFAT and myoD}

Nuclear translocation of MyoD and NFAT is a prerequisite for them to have transcriptional activity. Therefore, we examined the subcellular distribution of these transcription factors in hMSCs after PMA treatment. The results of Western blot analysis indicated that the amounts of NFAT and MyoD in the nuclear fraction increased with PMA treatment (Fig. 2a). However, the pattern of the increase was different. The nuclear localization of NFAT gradually increased from day 1 to day 3 and slightly decreased at day 6 in the PMA-treated group. Nevertheless, the amount of nuclear localized NFAT was still higher than that of the control group. On the other hand, the amount of nuclear MyoD increased on day 1 , and then decreased thereafter. This suggested that the effect of PMA may have been mediated mainly by NFAT rather than by MyoD.

We performed immunocytochemical staining using antibodies specific to the transcription factors. In untreated control hMSCs, both NFAT and MyoD were evenly distributed without prominent accumulation in the nucleus. However, when the cells were treated with PMA for 3 days, the amount of the transcription factors observed in the nucleus increased noticeably (Fig. 2b, c). To quantify the nuclear localization of both transcription factors, we further conducted a co-localization analysis for the transcription factors and DAPI-stained nuclei. The result indicated that PMA significantly increased the amount of both transcription factors that reside in the nucleus (Fig. 2d). The degree of colocalization was greater for NFAT than MyoD as evidenced by the higher PCC value. 

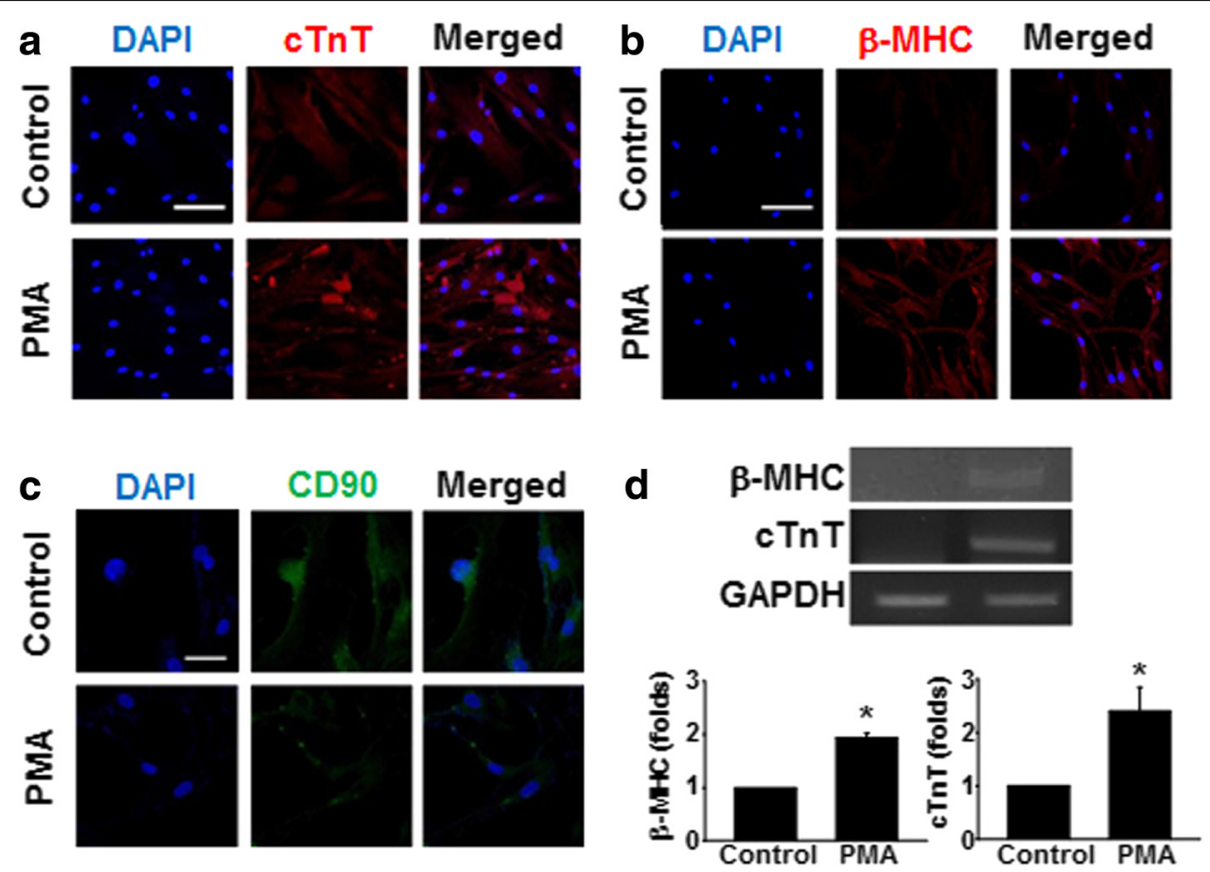

Fig. 1 Expression of cardiac and stem cell markers in PMA-treated hMSCs. Immunocytochemical analysis was performed to detect the expression of $\mathbf{a}$ CTnT (scale bar $=100 \mu \mathrm{m}), \mathbf{b} \beta-\mathrm{MHC}$ (scale bar $=100 \mu \mathrm{m})$, and $\mathbf{c}$ CD90 (scale bar $=40 \mu \mathrm{m})$ at 9 days after PMA $(1 \mu \mathrm{M})$ treatment. DAPI was used to stain the nucleus. $\mathbf{d}$ mRNA expression of $\beta-M H C$ and cTnT in hMSCs treated with PMA for 9 days was detected by RT-PCR. ${ }^{*} p<0.05$, versus control. cTnT cardiac troponin T, MHC myosin heavy chain, PMA phorbol 12-myristate 13-acetate

PMA increased calcineurin/calmodulin A interaction and ser9 phosphorylation of GSK3 $\beta$

The nuclear localization of NFAT was more pronounced than that of MyoD after PMA treatment, and this result may indicate that the effect of PMA was mainly mediated by NFAT. Thus, we further examined the underlying mechanisms of PMA with a focus on NFAT. Dephosphorylation of NFAT by phosphatases, such as calcineurin, and the subsequent nuclear translocation of NFAT increases its transcriptional activity [17]. Because the interaction with calmodulin facilitates the activation of calcineurin [18], we examined the interactions between calmodulin and calcineurin using the IP method. After $12 \mathrm{~h}$ of PMA $(1 \mu \mathrm{M})$ treatment, the interaction between calcineurin $\mathrm{A}$ and calmodulin increased compared with that of the control, suggesting that PMA induced calmodulin-dependent activation of calcineurin A (Fig. 3a). To examine whether myristoylated alanine-rich C kinase substrate (MARCKS) contributed to this PMAinduced interaction of calcineurin and calmodulin, we checked the effect of PMA on MARCKS phosphorylation. PMA increased phosphorylation of MARCKS up to the first $6 \mathrm{~h}$ and then the phosphorylation returned to baseline after $12 \mathrm{~h}$ (Fig. 3b), demonstrating that PMA-induced phosphorylation of MARCKS may have contributed to the increased calcineurin-calmodulin interaction. Another possible mechanism may involve inactivation of glycogen synthase kinase 3 beta (GSK3 $\beta$ ), which facilitates the phosphorylation of NFAT and subsequent nuclear exit $[19,20]$. According to our data, PMA increased the phosphorylation of GSK3 $\beta$ at serine 9, which indicated greater inactivation of GSK3 $\beta$ [21] compared with that of the untreated control (Fig. 3c). Although the amount of phosphorylated GSK3 $\beta$ in the cytoplasmic fraction peaked at day 1 and decreased thereafter, it was still pronounced until day 6 compared with the control.

\section{PMA induced the transcriptional activity of NFAT}

We constructed a GFP vector containing the NFAT binding motif of human interleukin-2 promoter (pAcNFAT-GFP; Additional file 1: Figure S1A). When pAcNFAT-GFP (500 ng/well, six-well plate)-transfected Hela cells were treated with $1 \mu \mathrm{M}$ PMA for $24 \mathrm{~h}$, the expression of GFP was pronounced compared with that of vehicle (DMSO)-treated pAc-NFAT-GFP-transfected cells (Additional file 1: Figure S1B). This result suggested that PMA induced the nuclear translocation and subsequent transcriptional activation of NFAT.

PMA induced p27 upregulation and proliferation arrest Temporal antagonism between cell cycle exit and differentiation has been demonstrated in cultured cells and animal models [22]. Discovered as an inhibitor of cyclin E/CDK2 (cyclin-dependent kinase 2) [23], the cyclindependent kinase inhibitor $1 \mathrm{~B}\left(\mathrm{p} 27^{\mathrm{kip} 1}\right)$ is a cell cycle inhibitor protein that stops or slows down the cell division 


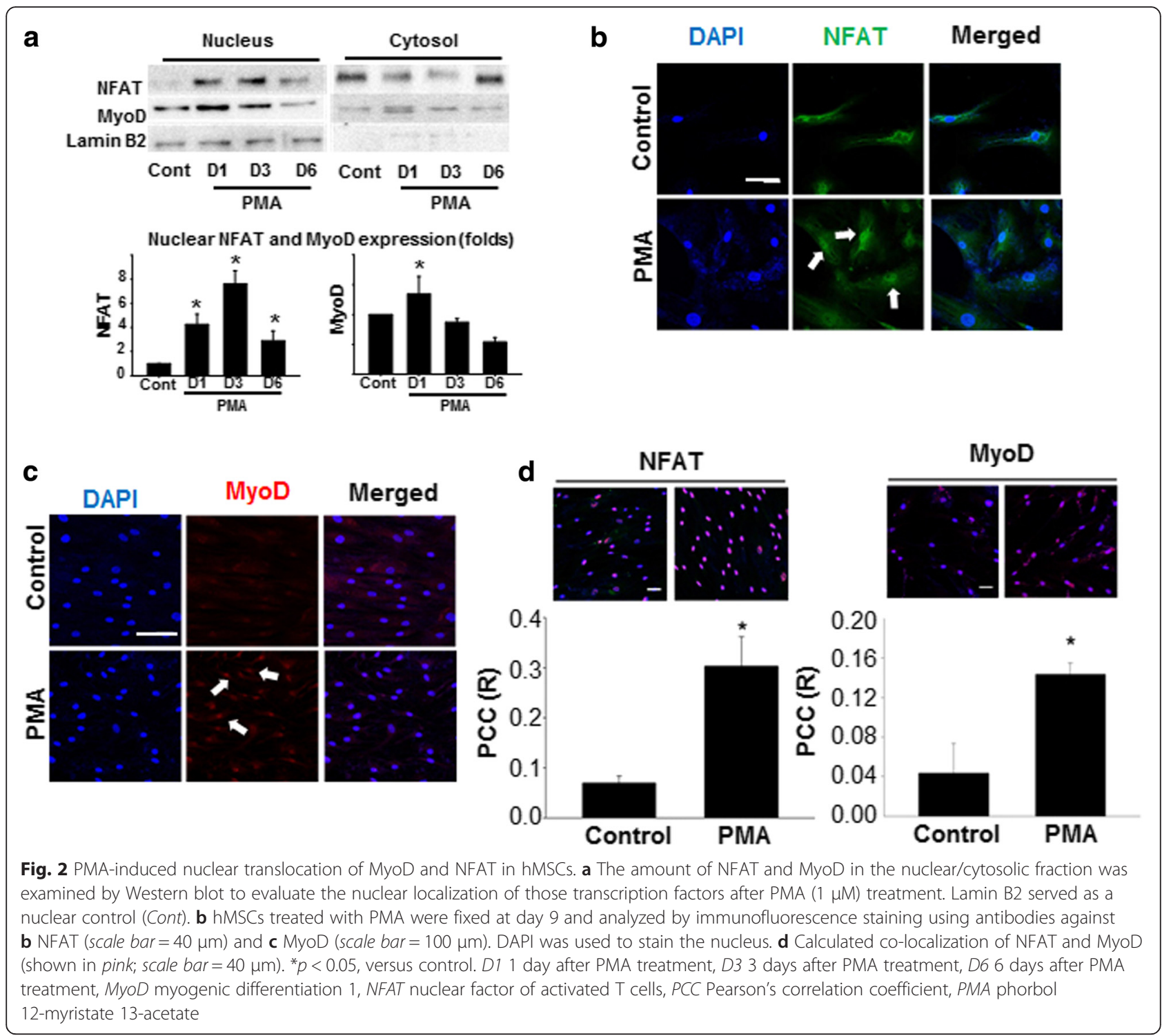

cycle. Because NFAT-mediated regulation of $\mathrm{p} 27^{\mathrm{kip} 1}$ has been reported [24, 25], we also examined the effect of PMA on the expression of $\mathrm{p} 27^{\mathrm{kip} 1}$ and cellular proliferation. Our data indicated that PMA treatment increased the expression of p27 $7^{\mathrm{kip} 1}$ at both the RNA (Fig. 4a) and protein (Fig. 4b) levels. Furthermore, PMA significantly decreased the expression of proliferating cell nuclear antigen (PCNA), suggesting that PMA induced cell cycle arrest in hMSCs (Fig. 4c).

\section{Discussion}

Here we demonstrated that NFAT is a key factor that mediates PMA-induced cardiac differentiation of hMSCs. In resting cells, the NFAT regulatory domain is phosphorylated by different kinases, including GSK3 [26-28]. This phosphorylation of the NFAT is important for the activation of NFAT because the phosphorylation status facilitates NFAT nuclear translocation [29]. Multiphosphorylation at this domain masks the nuclear localization sequence (NLS) of NFAT, resulting in its cytoplasmic localization [30]. Therefore, either promoting the dephosphorylation of NFAT or suppressing its phosphorylation, or both, can lead to nuclear translocation and subsequent transcriptional activation of NFAT. Our data suggest that PMA is involved in both of these mechanisms.

PMA is an activator of PKC [31], and activated PKC can both stimulate dephosphorylation and inhibit phosphorylation of NFAT. First, the PKC-dependent stimulation of NFAT dephosphorylation can be achieved by regulating calmodulin-calcineurin interactions. MARCKS is a PKC substrate that has a calmodulin-binding domain [32], and it is reportedly expressed in MSCs [33]. Upon phosphorylation by PKC, MARCKS dissociates from 


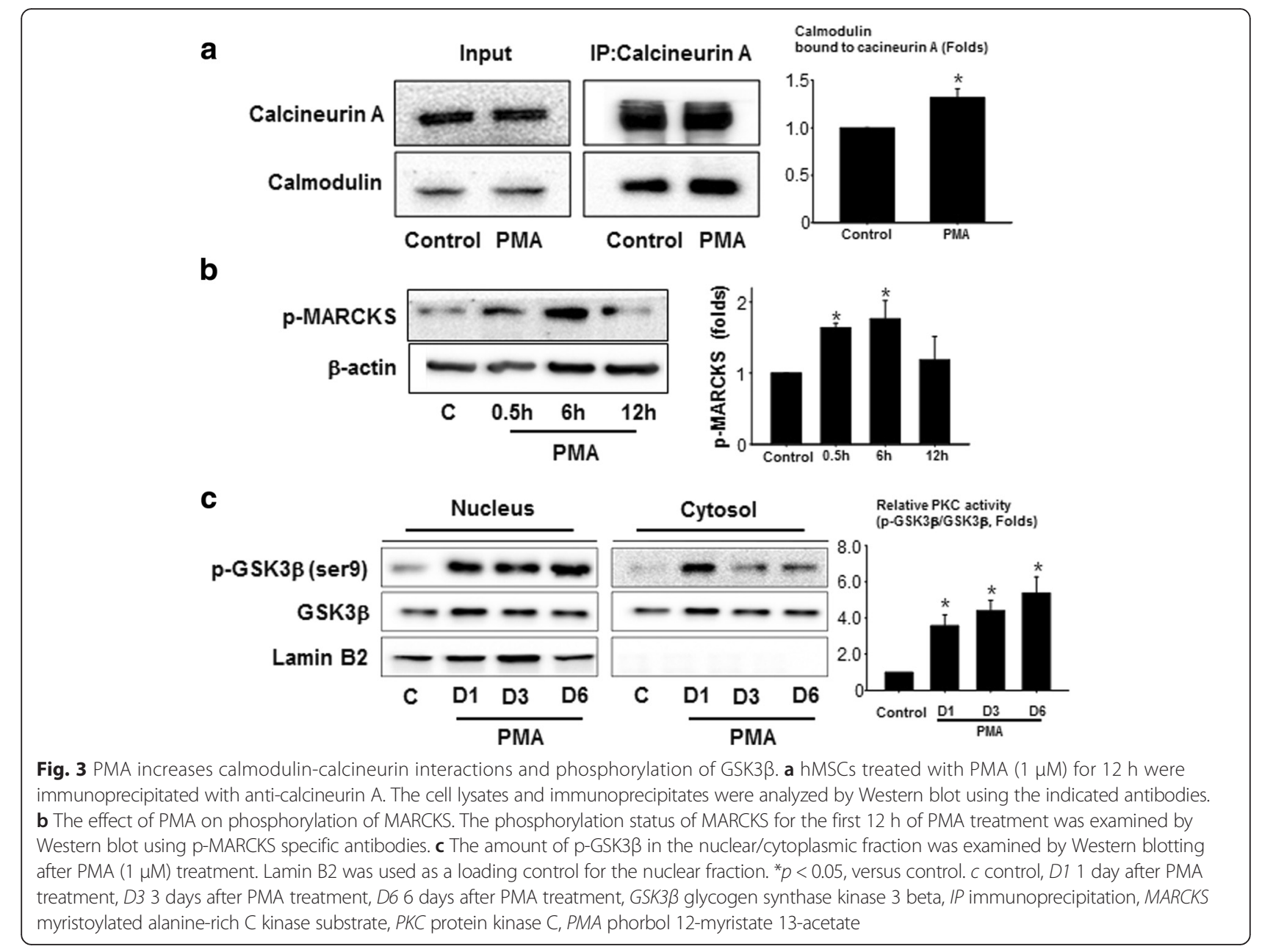

Calmodulin

calmodulin [34]. This result suggests that phosphorylation of MARCKS by PKC can lead to a significant increase in the local concentration of 'free' calmodulin that can bind to calcineurin (Fig. 4d). Because binding calmodulin allows calcineurin to exert its phosphatase activity [18], the activation of PKC by PMA may lead to the dephosphorylation of NFAT via the phosphorylation of MARCKS and a subsequent calmodulin/calcineurin interaction. In fact, our data indicated that PMA induced phosphorylation of MARCKS supporting MARCKS-mediated calcineurin/calmodulin interaction.

On the other hand, the activation of PKC may result in the suppression of NFAT phosphorylation by modulating GSK3 $\beta$ activity. Our data clearly demonstrated that PMA induced the phosphorylation of GSK3 $\beta$ (Fig. 3b), which is most likely mediated by PMAactivated PKC [35]. One thing should be noted: there was a transient upregulation of GSK3 $\beta$ after PMA treatment, with unknown mechanisms. Even after an extensive literature search the exact mechanism could not be found. However, since the activity of GSK3 $\beta$ is mainly regulated by its phosphorylation rather than expression itself, as long as PMA treatment increased the phosphorylation of GSK3 $\beta$, transient upregulation of GSK3 $\beta$ would not have significantly affected the result of this study.

Because the phosphorylation of GSK3 $\beta$ at Ser9 inhibits the kinase activity of GSK3 $\beta$ [21], the PMA-induced phosphorylation of GSK3 $\beta$ may decrease the possibility for NFAT phosphorylation by GSK3 $\beta$. In turn, the decreased phosphorylation of NFAT results in the increased transcriptional activity of NFAT (Fig. 4d). Although it is difficult to state that NFAT is the single most important transcription factor driving PMAmediated cardiac differentiation of MSCs, our study provides sufficient evidence that PMA activates the transcriptional activity of NFAT and a logical explanation for how PMA treatment leads to the transcriptional activation of NFAT. Although the GSK3 $\beta$ pathway and calmodulin pathway are discussed separately, there is also high possibility that those two pathways interact at some point to concert the effect of PMA. As far as we know, there is no previous study that has either proved or disproved GSK3 $\beta$ and calmodulin (or calcineurin) 
a
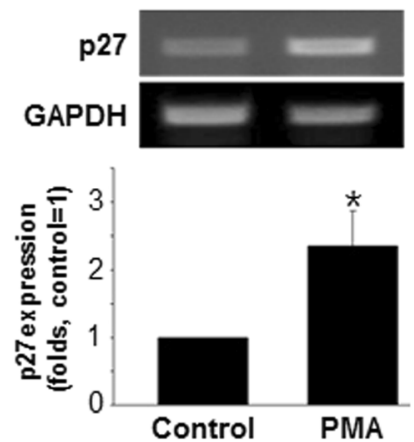

C

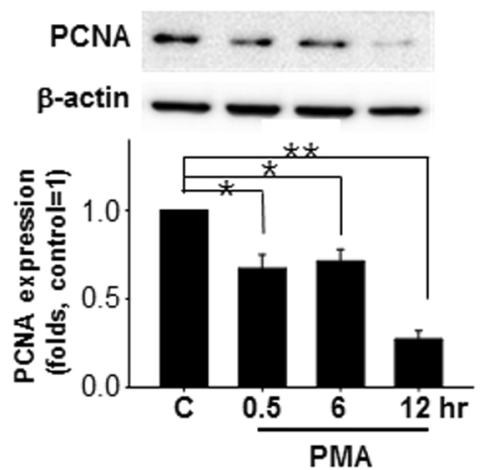

b

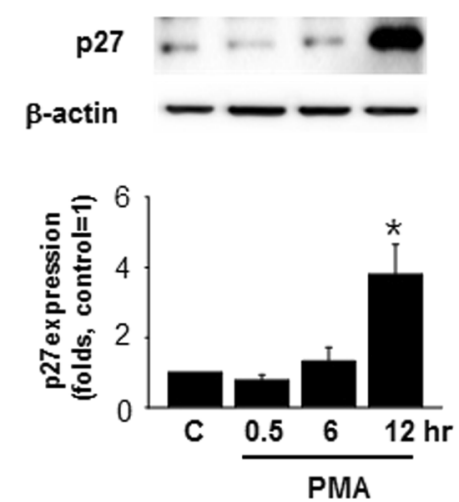

d

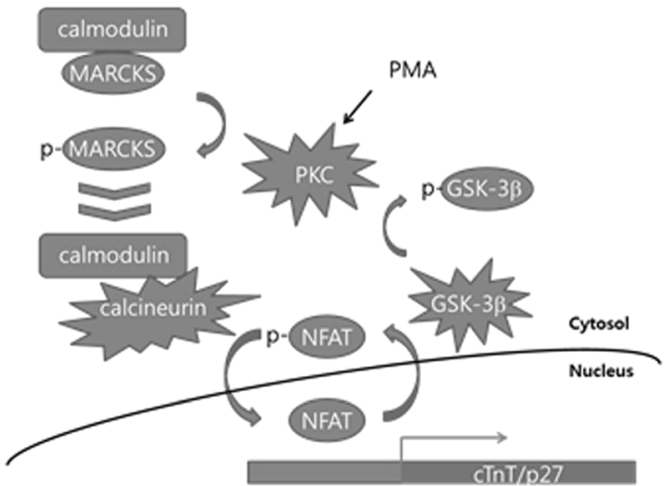

Fig. 4 PMA enhances p27 expression in hMSCs. a hMSCs were treated with PMA (1 $\mu$ M) for $12 \mathrm{~h}$. The expression of p2 $7^{\text {kip } 1}$ (p27) mRNA was measured by RT-RCR. $\mathbf{b}$ The p27 protein expression level was examined by Western blotting. The cells were treated with $1 \mu \mathrm{M}$ PMA for up to $12 \mathrm{~h}$. $\mathbf{c}$ PCNA expression was detected by Western blotting. $\mathbf{d}$ Schematic drawings of PMA-activated signaling pathways that lead to NFAT activation. ${ }^{*} p<0.05$, ${ }^{* *} p<0.01$, versus control. GSK3 $\beta$ glycogen synthase kinase 3 beta, MARCKS myristoylated alanine-rich $C$ kinase substrate, NFAT nuclear factor of activated T cells, PCNA proliferating cell nuclear antigen, PKC protein kinase C, PMA phorbol 12-myristate 13-acetate

interaction. Therefore, it would make a very interesting subject for future study.

Small molecules have great potential in controlling stem cell fate by regulating cellular processes such as proliferation, reprogramming and differentiation [36, 37]. In fact, a number of small molecules have been utilized to differentiate stem cells into cardiomyocytes [4-8], and it is expected that more small molecules will be tested for their abilities to regulate stem cell differentiation. Therefore, it is important to understand how small molecules induce stem cell differentiation because elucidating the underlying mechanism enables us to develop a strategy for synthesizing novel and improved differentiation-inducing small molecules. From that point of view, our study provides valuable information on the cardiac differentiation ability of PMA.

\section{Conclusion}

Our data demonstrated that the PKC activator PMA induced transcriptional activity of NFAT via both GSK3 $\beta$-mediated pathway and calcineurin/calmodulin- mediated pathway, leading to cardiac-specific marker expression. The results of this study recapitulate the importance of transcriptional regulation during MSC differentiation and help us to better understand the underlying mechanisms of small molecule-mediated MSC differentiation.

\section{Additional file}

Additional file 1: PMA increases the transcriptional activity of NFAT. (PDF $168 \mathrm{~kb}$ )

\section{Abbreviations}

BMP, bone morphogenetic protein; CDK, cyclin-dependent kinase; cTnT, cardiac troponin T; GFP, green fluorescent protein; GSK3 $\beta$, glycogen synthase kinase 3 beta; hMSC, human mesenchymal stem cell; IP, immunoprecipitation; MAPK, mitogen-activated protein kinase; MARCKS, myristoylated alanine-rich C kinase substrate; MHC, myosin heavy chain; MSC, mesenchymal stem cell; MyoD, myogenic differentiation 1; NFAT, nuclear factor of activated T cells; PCC, Pearson's correlation coefficient; PKC, protein kinase C; PMA, phorbol 12-myristate 13-acetate; RT-PCR, reverse transcription polymerase chain reaction 


\section{Acknowledgments}

This study was supported by grants funded by the Korea Ministry of Science, ICT and Future Planning (NRF-2015M3A9E6029519, and NFR2015M3A9E6029407).

\section{Authors' contributions}

HHS conceived the study, participated in all the experiments conducted, and revised the manuscript for important intellectual content. CYL and JL participated in cell culture and in vitro testing, and made significant contributions to revising the manuscript. SYL and EC provided technical assistance, supervised the experiments, and made significant contributions to revising the manuscript. JCP participated in the study design and provided technical assistance, and made significant contributions to revising the manuscript. SL participated in the study design, prepared the manuscript, and provided technical assistance. KCH conceived the study, participated in the study design and drafted and edited the manuscript. All authors read and approved the final manuscript.

\section{Competing interests}

The authors declare they have no competing interests.

\section{Author details}

'Brain Korea 21 PLUS Project for Medical Science, Yonsei University, Seoul, South Korea. ${ }^{2}$ Department of Integrated Omics for Biomedical Sciences, Yonsei University, Seoul, South Korea. ${ }^{3}$ Institute for Bio-medical Convergence, Catholic Kwandong University, Incheon, South Korea. ${ }^{4}$ Cellbiocontrol Laboratory, Department of Medical Engineering, Yonsei University College of Medicine, Seoul, South Korea. ${ }^{5}$ Department of Biomedical Sciences, College of Medicine, Catholic Kwandong University, Gangneung, Gangwon-do, South Korea.

Received: 18 March 2016 Revised: 3 June 2016

Accepted: 17 June 2016 Published online: 12 July 2016

\section{References}

1. Wei X, Yang X, Han ZP, Qu FF, Shao L, Shi YF. Mesenchymal stem cells: a new trend for cell therapy. Acta Pharmacol Sin. 2013;34(6):747-54. doi:10.1038/aps.2013.50.

2. Chang MG, Tung L, Sekar RB, Chang CY, Cysyk J, Dong P, et al. Proarrhythmic potential of mesenchymal stem cell transplantation revealed in an in vitro coculture model. Circulation. 2006;113(15):1832-41. doi:10.1161/CIRCULATIONAHA.105.593038.

3. Antonitsis P, loannidou-Papagiannaki E, Kaidoglou A, Papakonstantinou C. In vitro cardiomyogenic differentiation of adult human bone marrow mesenchymal stem cells. The role of 5-azacytidine. Interact Cardiovasc Thorac Surg. 2007;6(5):593-7. doi:10.1510/icvts.2007.157875.

4. Graichen R, Xu X, Braam SR, Balakrishnan T, Norfiza S, Sieh S, et al. Enhanced cardiomyogenesis of human embryonic stem cells by a small molecular inhibitor of p38 MAPK. Differentiation. 2008;76(4):357-70. doi:10.1111/j.14320436.2007.00236.x.

5. Yu PB, Hong CC, Sachidanandan C, Babitt JL, Deng DY, Hoyng SA, et al. Dorsomorphin inhibits BMP signals required for embryogenesis and iron metabolism. Nat Chem Biol. 2008:4(1):33-41. doi:10.1038/nchembio.2007.54.

6. Ren Y, Lee MY, Schliffke S, Paavola J, Amos PJ, Ge X, et al. Small molecule Wnt inhibitors enhance the efficiency of BMP-4-directed cardiac differentiation of human pluripotent stem cells. J Mol Cell Cardiol. 2011; 51(3):280-7. doi:10.1016/j.yjmcc.2011.04.012.

7. Willems E, Spiering S, Davidovics H, Lanier M, Xia Z, Dawson M, et al. Small-molecule inhibitors of the Wnt pathway potently promote cardiomyocytes from human embryonic stem cell-derived mesoderm. Circ Res. 2011:109(4):360-4. doi:10.1161/CIRCRESAHA.111.249540.

8. Wang $\mathrm{H}$, Hao J, Hong CC. Cardiac induction of embryonic stem cells by a small molecule inhibitor of Wnt/beta-catenin signaling. ACS Chem Biol. 2011;6(2):192-7. doi:10.1021/cb100323z

9. Song H, Hwang HJ, Chang W, Song BW, Cha MJ, Kim IK, et al. Cardiomyocytes from phorbol myristate acetate-activated mesenchymal stem cells restore electromechanical function in infarcted rat hearts. Proc Natl Acad Sci U S A. 2011;108(1):296-301. doi:10.1073/pnas.1015873107.

10. Seward DJ, Haney JC, Rudnicki MA, Swoap SJ. bHLH transcription factor MyoD affects myosin heavy chain expression pattern in a muscle-specific fashion. Am J Physiol Cell Physiol. 2001;280(2):C408-13.
11. Weintraub H, Tapscott SJ, Davis RL, Thayer MJ, Adam MA, Lassar AB, et al. Activation of muscle-specific genes in pigment, nerve, fat, liver, and fibroblast cell lines by forced expression of MyoD. Proc Natl Acad Sci U S A. 1989:86(14):5434-8.

12. Brunelli S, Relaix F, Baesso S, Buckingham M, Cossu G. Beta cateninindependent activation of MyoD in presomitic mesoderm requires PKC and depends on Pax3 transcriptional activity. Dev Biol. 2007;304(2):604-14. doi:10.1016/j.ydbio.2007.01.006

13. Schubert W, Yang XY, Yang TT, Factor SM, Lisanti MP, Molkentin JD, et al. Requirement of transcription factor NFAT in developing atrial myocardium J Cell Biol. 2003;161(5):861-74. doi:10.1083/jcb.200301058.

14. San-Antonio B, Iniguez MA, Fresno M. Protein kinase Czeta phosphorylates nuclear factor of activated T cells and regulates its transactivating activity. J Biol Chem. 2002;277(30):27073-80. doi:10.1074/jbc.M106983200.

15. Dunn KW, Kamocka MM, McDonald JH. A practical quide to evaluating colocalization in biological microscopy. Am J Physiol Cell Physiol. 2011;300(4):C723-42. doi:10.1152/ajpcell.00462.2010.

16. Granelli-Piperno A, McHugh P. Characterization of a protein that regulates the DNA-binding activity of NF-AT, the nuclear factor of activated T cells. Proc Natl Acad Sci U S A. 1991;88(24):11431-4.

17. Hogan PG, Chen L, Nardone J, Rao A. Transcriptional regulation by calcium, calcineurin, and NFAT. Genes Dev. 2003;17(18):2205-32. doi:10.1101/gad.1102703.

18. Ye Q, Feng Y, Yin Y, Faucher F, Currie MA, Rahman MN, et al. Structural basis of calcineurin activation by calmodulin. Cell Signal. 2013;25(12):2661-7. doi:10.1016/j.cellsig.2013.08.033.

19. Beals CR, Sheridan CM, Turck CW, Gardner P, Crabtree GR. Nuclear export of NF-ATc enhanced by glycogen synthase kinase-3. Science. 1997;275(5308):1930-4.

20. Qu Z, Sun D, Young W. Lithium promotes neural precursor cell proliferation: evidence for the involvement of the non-canonical GSK-3beta-NF-AT signaling. Cell Biosci. 2011;1(1):18. doi:10.1186/2045-3701-1-18.

21. Shaw M, Cohen P, Alessi DR. Further evidence that the inhibition of glycogen synthase kinase-3beta by IGF-1 is mediated by PDK1/PKB-induced phosphorylation of Ser-9 and not by dephosphorylation of Tyr-216. FEBS Lett. 1997:416(3):307-11.

22. Ruijtenberg S, van den Heuvel S. Coordinating cell proliferation and differentiation: antagonism between cell cycle regulators and cell type-specific gene expression. Cell Cycle. 2016;15(2):196-212. doi:10.1080/15384101.2015.1120925

23. Slingerland JM, Hengst L, Pan CH, Alexander D, Stampfer MR, Reed SI. A novel inhibitor of cyclin-Cdk activity detected in transforming growth factor beta-arrested epithelial cells. Mol Cell Biol. 1994;14(6):3683-94

24. Wang Q, Zhou Y, Jackson LN, Johnson SM, Chow CW, Evers BM. Nuclear factor of activated T cells (NFAT) signaling regulates PTEN expression and intestinal cell differentiation. Mol Biol Cell. 2011;22(3):412-20. doi:10.1091/ mbc.E10-07-0598.

25. Santini MP, Talora C, Seki T, Bolgan L, Dotto GP. Cross talk among calcineurin, Sp1/Sp3, and NFAT in control of p21(WAF1/CIP1) expression in keratinocyte differentiation. Proc Natl Acad Sci U S A. 2001;98(17):9575-80. doi:10.1073/pnas.161299698.

26. Okamura H, Garcia-Rodriguez C, Martinson H, Qin J, Virshup DM, Rao A. A conserved docking motif for CK1 binding controls the nuclear localization of NFAT1. Mol Cell Biol. 2004;24(10):4184-95.

27. Neal JW, Clipstone NA. Glycogen synthase kinase-3 inhibits the DNA binding activity of NFATC. J Biol Chem. 2001;276(5):3666-73. doi:10.1074/jbc. M004888200.

28. Chow W, Hou G, Bendeck MP. Glycogen synthase kinase 3beta regulation of nuclear factor of activated T-cells isoform $\mathrm{C} 1$ in the vascular smooth muscle cell response to injury. Exp Cell Res. 2008;314(16):2919-29. doi:10.1016/j. yexcr.2008.07.010

29. Okamura H, Aramburu J, Garcia-Rodriguez C, Viola JP, Raghavan A, Tahiliani $\mathrm{M}$, et al. Concerted dephosphorylation of the transcription factor NFAT1 induces a conformational switch that regulates transcriptional activity. Mol Cell. 2000;6(3):539-50.

30. Rao A, Luo C, Hogan PG. Transcription factors of the NFAT family: regulation and function. Annu Rev Immunol. 1997;15:707-47. doi:10.1146/annurev. immunol.15.1.707.

31. Castagna M, Takai Y, Kaibuchi K, Sano K, Kikkawa U, Nishizuka Y. Direct activation of calcium-activated, phospholipid-dependent protein kinase by tumor-promoting phorbol esters. J Biol Chem. 1982;257(13):7847-51. 
32. Yamauchi E, Nakatsu T, Matsubara M, Kato H, Taniguchi H. Crystal structure of a MARCKS peptide containing the calmodulin-binding domain in complex with Ca2 +-calmodulin. Nat Struct Biol. 2003;10(3):226-31. doi:10. 1038/nsb900.

33. Miller JD, Lankford SM, Adler KB, Brody AR. Mesenchymal stem cells require MARCKS protein for directed chemotaxis in vitro. Am J Respir Cell Mol Biol. 2010;43(3):253-8. doi:10.1165/rcmb.2010-0015RC.

34. Verghese GM, Johnson JD, Vasulka C, Haupt DM, Stumpo DJ, Blackshear PJ. Protein kinase C-mediated phosphorylation and calmodulin binding of recombinant myristoylated alanine-rich $C$ kinase substrate (MARCKS) and MARCKS-related protein. J Biol Chem. 1994;269(12):9361-7.

35. Fang X, Yu S, Tanyi JL, Lu Y, Woodgett JR, Mills GB. Convergence of multiple signaling cascades at glycogen synthase kinase 3: Edg receptor-mediated phosphorylation and inactivation by lysophosphatidic acid through a protein kinase C-dependent intracellular pathway. Mol Cell Biol. 2002;22(7):2099-110.

36. Yu C, Liu K, Tang S, Ding S. Chemical approaches to cell reprogramming. Curr Opin Genet Dev. 2014;28:50-6. doi:10.1016/j.gde.2014.09.006.

37. Xu Y, Shi Y, Ding S. A chemical approach to stem-cell biology and regenerative medicine. Nature. 2008;453(7193):338-44. doi:10.1038/ nature07042.

Submit your next manuscript to BioMed Central and we will help you at every step:

- We accept pre-submission inquiries

- Our selector tool helps you to find the most relevant journal

- We provide round the clock customer support

- Convenient online submission

- Thorough peer review

- Inclusion in PubMed and all major indexing services

- Maximum visibility for your research

Submit your manuscript at www.biomedcentral.com/submit
) Biomed Central 